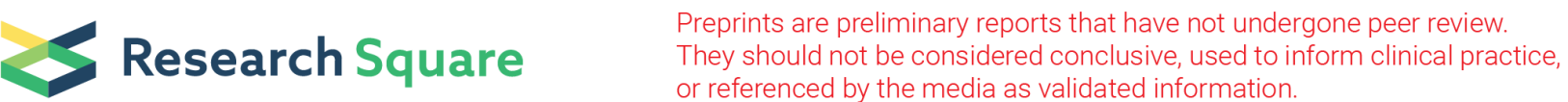

\section{Description of an Integrated and Dynamic System to Efficiently Deal with a Raging COVID-19 Pandemic Peak}

Vanni Agnoletti ( $\nabla$ vanni.agnoletti@auslromagna.it)

AUSL della Romagna: Azienda Unita Sanitaria Locale della Romagna https://orcid.org/0000-0002-70932749

\section{Emiliano Gamberini}

AUSL della Romagna: Azienda Unita Sanitaria Locale della Romagna Alessandro Circelli

AUSL della Romagna: Azienda Unita Sanitaria Locale della Romagna Costanza Martino

AUSL della Romagna: Azienda Unita Sanitaria Locale della Romagna Domenico Pietro Santonastaso

AUSL della Romagna: Azienda Unita Sanitaria Locale della Romagna

\section{Giuliano Bolondi}

AUSL della Romagna: Azienda Unita Sanitaria Locale della Romagna

\section{Giorgia Bastoni}

AUSL della Romagna: Azienda Unita Sanitaria Locale della Romagna

\section{Martina Spiga}

AUSL della Romagna: Azienda Unita Sanitaria Locale della Romagna Paola Ceccarelli

AUSL della Romagna: Azienda Unita Sanitaria Locale della Romagna Luca Montaguti

AUSL della Romagna: Azienda Unita Sanitaria Locale della Romagna

\section{Fausto Catena}

AUSL della Romagna: Azienda Unita Sanitaria Locale della Romagna

Carlo Lusenti

AUSL della Romagna: Azienda Unita Sanitaria Locale della Romagna

\section{Mattia Altini}

AUSL della Romagna: Azienda Unita Sanitaria Locale della Romagna Emanuele Russo

AUSL della Romagna: Azienda Unita Sanitaria Locale della Romagna 
Keywords: COVID-19, Intensive Care Unit, High-dependency Unit, Step-down Unit, Hospital Admission Criteria, Bed management, Bed occupancy rate, Patients throughput

Posted Date: May 26th, 2021

DOI: https://doi.org/10.21203/rs.3.rs-528572/v1

License: (c) (i) This work is licensed under a Creative Commons Attribution 4.0 International License. Read Full License

Version of Record: A version of this preprint was published at Frontiers in Medicine on March 18th, 2022. See the published version at https://doi.org/10.3389/fmed.2022.819134. 


\section{Abstract}

\section{Background}

To describe an innovative and functional method to deal with the increased COVID-19 pandemic-related intensive care unit bed requirements.

\section{Methods}

We describe the emergencial creation of integrated system of internistic ward, step-down unit and intensive care unit, physically located in reciprocal vicinity at the same floor. The run under the control of a single intensive care staff, sharing clinical protocols and informatic system, following a single director supervision. The intention was to create a dynamic and flexible system, allowing for rapid and fluid patient admission/discharge, depending on the requirements due to the third Italian peak of COVID-19 pandemic in March 2021.

\section{Results}

142 COVID-19 patients and 66 non-COVID-19 patients were admitted, no critical patient was left unadmitted and no COVID-19 severe patients referring to our centre had to be redirected to other hospitals due to bed saturation. This system allowed shorter hospital length-of-stay in general wards (5.9 \pm 4 days) than in other internistic COVID-19 wards and an overall mortality in line with those reported in literature despite the peak raging.

\section{Conclusion}

This case report shows the feasibility and the efficiency of this dynamic model of hospital rearrangement to deal with COVID-19 pandemic peaks.

\section{Introduction}

COVID-19 pandemic has pressured healthcare systems worldwide. We previously described a functional and dynamic strategy that allowed our intensive care unit (ICU) to deal with the first two peaks of pandemic, in March and October 2020, that in our community reached 8.2 cases per 1,000 inhabitants. ${ }^{1}$

The setting is a public hospital of 450 beds, reference point for a population of about 210,000 people. Before COVID-19, the ICU consisted of 18 beds; during the pandemic, 6 non-COVID ICU beds and 9 stepdown unit (SDU) beds were opened.

This case study aims to show how this dynamic system has been furtherly implemented to deal with the third and strongest pandemic peak, in March 2021, reaching a local incidence of 13,3 positive cases per 1,000 inhabitants (62\% increase).

\section{Methods}


As the third peak was arising, all the hospital non-COVID wards were promptly resized and relocated. Strategically, the first units left empty were those close to the ICU-SDU (4th floor) and to the COVID-internal medicine (6th floor).

With respect to the previous strategy, a further internistic COVID unit, General Ward Covid-19 (GW C19 $)$, of 16 beds was carved out at 4th floor, next to the SDU (Fig. 1). It was staffed by an intensivist physician, with a 8:1 patient-per-nurse ratio (PPNR); nurses were not ICU-trained. Two monitored beds (oxygen saturation, non invasive or invasive blood pressure and electrocardiogram) for patients needing high flow nasal cannulae (HFNC) were available. Admission criteria to this general ward were: patients positive to Severe acute respiratory syndrome coronavirus 2 (SARS-CoV-2), with radiologic evidence of pulmonary interstitiopathy, dyspnea, oxygen saturation $\left(\mathrm{SpO}_{2}\right) \geq 92 \%$ on room air, low oxygen supplementation (less $10 \mathrm{~L} / \mathrm{min}$ ). Clinical factors such as fever, hypertension and diabetes mellitus were considered risk factors for possibly severe evolution. ${ }^{2}$

The 4th floor, under the control of the intensive care department, thus counted on: $16 \mathrm{GW}_{\mathrm{C} 19}$ beds, 1 intensivist, 8:1 PPNR; 9 beds SDU, 1 intensivist, 3:1 PPNR (at least 1 ICU-trained nurse); 11 ICU1 beds, 2 intensivists (1 during night shift), 2:1 PPNR; 7 ICU2 beds, 2 intensivists (1 during night shift), 2:1 PPNR; 5 ICU3 beds, 1 intensivist, 2:1 PPNR (Fig. 1). Admission criteria to SDU were previously described. ${ }^{1}$

This was made possible by a drastic 50\% reduction of the planned operating room activity, actuated for safety reasons, that allowed to recover a sufficient number of anesthetists to be employed at 4th floor. In Italy, anesthesia and intensive care still constitute a single residency program, thus specialists are certified and trained to manage complex patients with one or more ongoing organ failure.

In March 2021, the Italian Ministry of Health published new guidelines on the management of isolation of severe COVID-19 patients: ${ }^{3} 21$ days after the first SARS-CoV-2 positivity, if asymptomatic or with a negative molecular test, they were considerable non-infective and transferable to non-COVID units. This increased the fluidity in the management of bed occupancy.

\section{Results}

In March 2021 (31 days), a total of 142 COVID-19 patients and 66 non-COVID-19 patients were admitted to the 4th floor. Table 1 describes the characteristics of these patients. The 4th floor setting allowed to admit every patient to a level of intensiveness appropriate for their clinical status.

Mean length-of-stay (LOS) (standard deviation - SD) in days was 5.9 (4.0) for $\mathrm{GW}_{\mathrm{C} 19}, 7.0$ (4.6) for SDU, 8.6 (6.7) for Intensive Care Unit Covid-19(ICU $\mathrm{C} 19)$ and 4.0 (5.3) for Intensive Care Unit No Covid-19 (ICU no-C19). The mortality rate was $2.6 \%$ for $\mathrm{GW}_{\mathrm{C} 19}, 20 \%$ for SDU, $36 \%$ for $\mathrm{ICU}_{\mathrm{C} 19}$ and $9.2 \%$ for $\mathrm{ICU}_{\text {no-C19, }}$, in line with available literature.

Table 2 and Fig. 2 summarize the overall flow of patients between different units. 
Mean ( \pm SD) LOS in $\mathrm{GW}_{\mathrm{C} 19}$ was shorter than in other internistic COVID-19 wards: 5.9 (4.0) days vs 11.8 (5.0) days. This is probably due to the higher rapidity by which patients were transferred to a higher level of care through early detection of clinical deterioration and simple transfer systems. All patients with severe COVID-19 who referred to our hospital have been hospitalized, none needed to be referred to other hospitals, thanks to a system that avoided hospital bed saturation.

\section{Discussion}

The decision to staff the $\mathrm{GW}_{\mathrm{C} 19}$ with anesthesiologists/intensivists was proposed by the hospital direction due to staff contingency. The director of anesthesia and intensive care unit and the collaborators agreed with this setting, in order to ease and improve the management of patient discharge from ICU and SDU, trying to avoid bed saturation. Being part of the same team, sharing the same protocols, informatic system (Margherita 3) and coordinators allowed considerable time saving. This was an efficient solution to maintain a safety and balanced hospital environment.

Differently from the previous report from March and April 2020, SDU worked more as a high dependency unit (HDU), at a semi-intensive care level, more complex than a common step-down-unit. ${ }^{4} 8$ patients were transferred from $\mathrm{ICU}_{\mathrm{C} 19}$ to SDU without requiring invasive ventilation; of the 41 patients admitted in SDU, only 11 needed escalation to $\mathrm{ICU}_{\mathrm{C} 19}$ for higher monitoring or orotracheal intubation requirements; 34 patients were admitted directly from the emergency department (ED) to SDU. These data seem to testify the high intensity of care reached in SDU at this third wave.

A limitation of this report is that, by its nature of case study, it is not matched with a comparative system. Moreover, at a first superficial sight, the employment of intensivists in $\mathrm{GW}_{\mathrm{C} 19}$ and SDU might seem a wastefulness of resources. In our experience, this has allowed many physicians to cyclically work at a lower intensity, periodically decompressing from the stress and pressure of a year in a ICU $\mathrm{C}_{19}$, interacting with conscious patients experiencing better outcomes, thus reducing burnout problem. ${ }^{5}$

A further limitation of this system is that it worked in our specific context: it might not be applicable for hospitals acting as referral centers for a much wider general population and it might also not be applicable to regions where the incidence rate is much higher, determining a dramatic pandemic wave.

The model of differential intensity for hospital care management (high-intensity for ICU, medium-intensity for SDU and low-intensity for GW), handled by a single intensive care unit, determined a sort of independence of the 4th floor from the hospital. The 4th floor was able to admit COVID-19 patients from other units/floors as needed from their clinical status evaluated by a consultant, but internally there was no need of hospital bed-manager coordination, counselling requests, bureaucracy and time-wasting procedures.

\section{Conclusion}


The use of a COVID-19 "critical floor" from general ward to ICU is an example of system adaptability. The effort made by intensivists was useful for patients in terms of quality of care and for doctors in terms of occupational stress and mental health.

\section{Abbreviations}

ICU: intensive care unit; SDU: step-down unit; PPNR: patient-per-nurse ratio; HFNC: high flow nasal cannulae; SARS-CoV-2: Severe acute respiratory syndrome coronavirus 2; $\mathrm{SpO}_{2}$ : oxygen saturation; $\mathrm{GW}_{\mathrm{C} 19}$ :

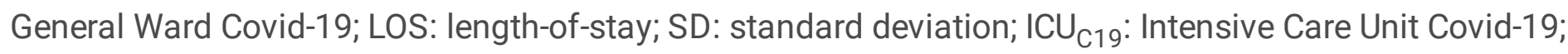

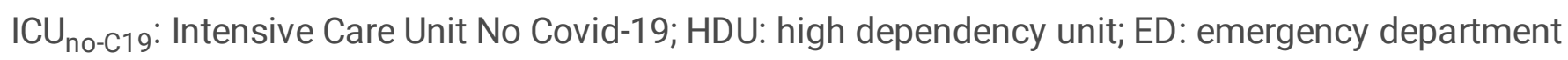

\section{Declarations}

Ethics approval and consent to participate: Not applicable

Consent for publication: Not applicable

Availability of data and materials: Anonymized datasets used and/or analyzed not including identifiable patient information are available from the corresponding author on reasonable request.

Competing interests: none

Funding: This research did not receive any specific grant from funding agencies in the public, commercial, or not-for-profit sectors.

\section{Authors' contributions}

VA conceived the study and wrote the article; EG, AC, CM, DPS, GB worked in Covid ICU and Wards and helped to write the article; GB, MS, PC coordinated the nursing staff in the management of the ward; LM, FC, CL, MA collaborated to review the manuscript; ER helped to write the manuscript and provided supervision

Acknowledgements: Authors thank all the colleagues (physicians, nurses and healthcare operators) who cannot be mentioned in this paper, who bravely and restlessly collaborated to face this year of COVID-19 pandemic.

\section{References}

1. Agnoletti V, Russo E, Circelli A, et al. From intensive care to step-down units: Managing patients throughput in response to COVID-19. Int J Qual Health Care. 2021 Feb;20(1):mzaa091. 33(.

2. Yetmar ZA, Issa M, Munawar S, et al. Inpatient Care of Patients with COVID-19: A Guide for Hospitalists. Am J Med. 2020 Sep;133(9):1019-24. 
3. COVID-19. indicazioni per la durata e il termine dell'isolamento e della quarantena, availableat:http://www.salute.gov.it/portale/nuovocoronavirus/dettaglioNotizieNuovoCoronavirus.jsp? lingua=italiano\&id $=5117$.

4. Solberg BC, Dirksen CD, Nieman FH, et al. Introducing an integrated intermediate care unit improves ICU utilization: a prospective intervention study. BMC Anesthesiol. 2014 Sep 6;14:76. doi: 10.1186/1471-2253-14-76. PMID: 25276092; PMCID: PMC4177684.

5. Magnavita N, Soave PM, Ricciardi W, et al Occupational Stress and Mental Health among Anesthetists during the COVID-19 Pandemic. Int J Environ Res Public Health. 2020 Nov 8;17(21):8245.

\section{Figures}

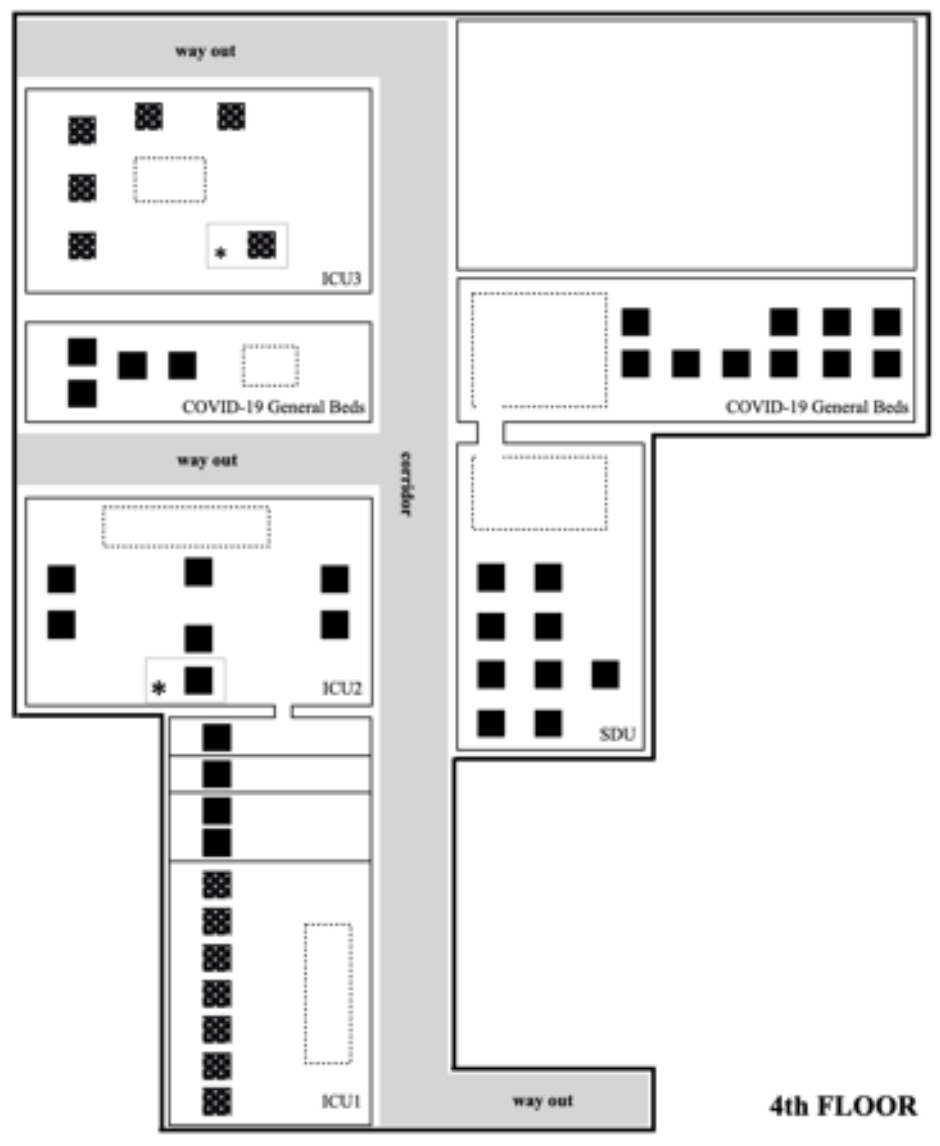

No Covid-19 ICU beds

Covid-19 ICU beds

Extra ICU bed No Covid-19

Extra ICU bed Covid-19

\section{Figure 1}

Critical Area at the 4th floor 


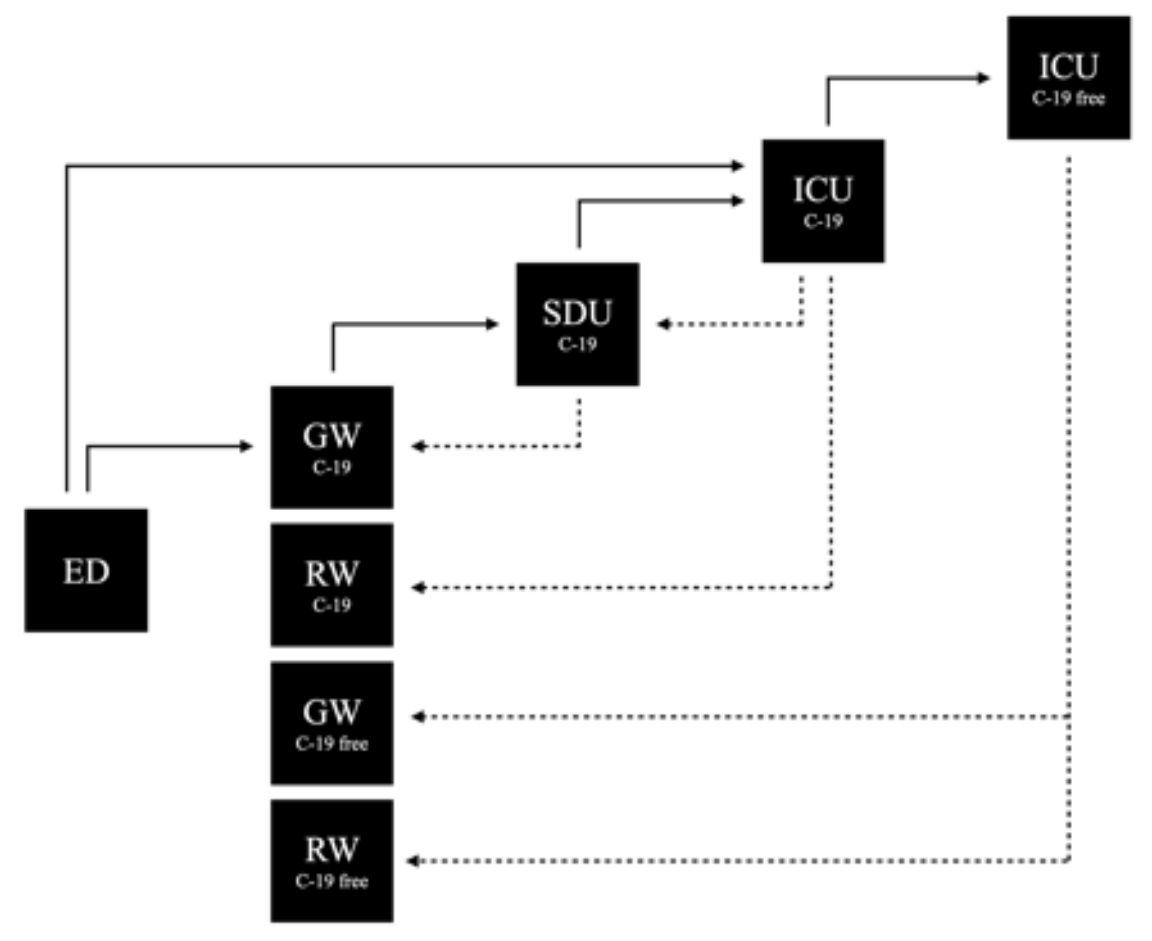

ED: Emergency Department

GW C-19: General Ward Covid-19

RW C-19: Rehabilitation Ward Covid-19

SDU C-19: Step Down Unit Covid-19

ICU C-19: Intensive Care Unit Covid-19

ICU C-19 free: Intensive Care Unit no-Covid-19

GW C-19 free: General Ward no-Covid-19

RW C-19 free: Rehabilitation Ward no-Covid-19

\section{Figure 2}

Patient Covid-19 flow ED: Emergency Department GW C-19: General Ward Covid-19 RW C-19: Rehabilitation Ward Covid-19 SDU C-19: Step Down Unit Covid-19 ICU C-19: Intensive Care Unit Covid-19 ICU C-19 free: Intensive Care Unit no-Covid-19 GW C-19 free: General Ward no-Covid-19 RW C-19 free: Rehabilitation Ward no-Covid-19 